\title{
Chitosan-Covered Pd@Pt Core-Shell Nanocubes for Direct Electron Transfer in Electrochemical Enzymatic Glucose Biosensor
}

\author{
Siva Kumar Krishnan, ${ }^{* \dagger}{ }^{\dagger}$ Evgen Prokhorov, ${ }^{\ddagger}$ Daniel Bahena, ${ }^{\S}$ Rodrigo Esparza, ${ }^{* \dagger}{ }^{\dagger}$ and M. Meyyappan \\ ${ }^{\dagger}$ Centro de Física Aplicada y Tecnología Avanzada, Universidad Nacional Autónoma de México, Boulevard Juriquilla 3001, Santiago \\ de Querétaro, Querétaro 76230, Mexico \\ ${ }^{\ddagger}$ Centro de Investigación y Estudios Avanzados, Unidad Querétaro, Santiago de Querétaro, Querétaro 76230, Mexico \\ ${ }^{\S}$ Advanced Laboratory of Electron Nanoscopy, Cinvestav, Av. Instituto Politecnico Nacional, 2508, Col. San Pedro Zacatenco, \\ Delegacion Gustavo A. Madero, Mexico D.F. CP 07360, Mexico \\ "Center for Nanotechnology, NASA Ames Research Center, Moffett Field, Mountain View, California 94035, United States
}

Supporting Information

\begin{abstract}
Development of biosensors with high sensitivity, high spatial resolution, and low cost has received significant attention for their applications in medical diagnosis, diabetes management, and environment-monitoring. However, achieving a direct electrical contact between redox enzymes and electrode surfaces and enhancing the operational stability still remain as challenges. Inorganic metal nanocrystals (NCs) with precisely controlled shape and surface structure engineered with an appropriate organic coating can help overcome the challenges associated with their stability and aggregation for practical biosensor applications. Herein, we describe a facile, room-temperature, seed-mediated solution-phase route to

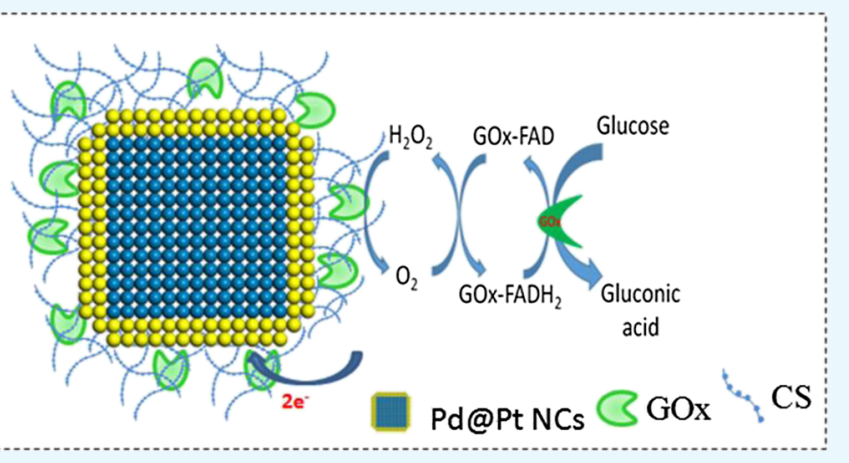
synthesize monodisperse Pd@Pt core-shell nanocubes with subnanometer-thick platinum $(\mathrm{Pt})$ shells. The enzyme electrode consisting of Pd@Pt core-shell NCs was first covered with a chitosan (CS) polymer and then glucose oxidase (GOx) immobilized by a covalent linkage to the CS. This polymer permits covalent immobilization through active amino $(-\mathrm{NH})$ side groups to improve the stability and preserve the biocatalytic functions while the Pd@Pt NCs facilitate enhanced direct electron transfer (DET) in the biosensor. The resultant biosensor promotes DET and exhibits excellent performance for the detection of glucose, with a sensitivity of $6.82 \mu \mathrm{A} \mathrm{cm}{ }^{-2} \mathrm{mM}^{-1}$ and a wide linear range of 1-6 mM. Our results demonstrate that sensitive electrochemical glucose detection based on Pd@Pt core-shell NCs provides remarkable opportunities for designing low-cost and sensitive biosensors.
\end{abstract}

\section{INTRODUCTION}

Metal nanocrystals (NCs) with distinct shapes and controllable facets have received substantial attention because of their attractive properties such as enhanced electrocatalytic activity and biosensing properties. ${ }^{1,2}$ Recently, development of platinum (Pt)-based bimetallic nanostructures with tailored geometries (e.g., core-shell nanostructures with ultrathin $\mathrm{Pt}$ shell thickness) has received attention toward enabling enhanced electrocatalytic activity and reducing cost. ${ }^{1,3}$ In particular, Pd@Pt nanostructures have been extensively reported for various applications such as fuel cell electrodes, ${ }^{3}$ hydrogen peroxide $\left(\mathrm{H}_{2} \mathrm{O}_{2}\right)$ and glucose biosensing, ${ }^{4-6}$ and gas sensors. ${ }^{7}$ Also, Pd has been widely used as a substrate to deposit Pt layers because of its very similar lattice constant and chemical stability. ${ }^{8}$ Ultrathin $\mathrm{Pt}$ layers on single-crystal substrates have been prepared by vacuum deposition. ${ }^{9}$ The seed-mediated solution-phase technique has been widely used recently for the deposition of Pt-on-Pd NCs for the Pd@Pt core-shell nanostructures, wherein thickness of the Pt shell can be controlled at 1-6 atomic layers. ${ }^{10,11}$ However, precise control of the thickness of the $\mathrm{Pt}$ layers down to the subnanometer level is difficult because of the galvanic replacement reaction between the two metals in aqueous solutions, which leads to the formation of voids (small holes) or concave structures at room temperature. ${ }^{3}$ In addition, the high intrinsic surface energy and interatomic bond energy (307 kJ/ mol) of Pt lead to selective deposition at the corner sites typically adopts an island growth mode (Volmer-Weber mode $)^{12}$ and followed by the diffusion onto the other faces of the NCs. Thus, uniform deposition of Pt can be achieved only at a higher reaction temperature by altering the diffusion rates. ${ }^{10}$ More recently, Yang et al. ${ }^{13}$ have reported the galvanic replacement-free deposition of an ultrathin shell of $0.6 \mathrm{~nm}$ thick $\mathrm{Au}$ on Ag nanocubes. The galvanic reaction was avoided by

Received: January 18, 2017

Accepted: April 26, 2017

Published: May 8, 2017 

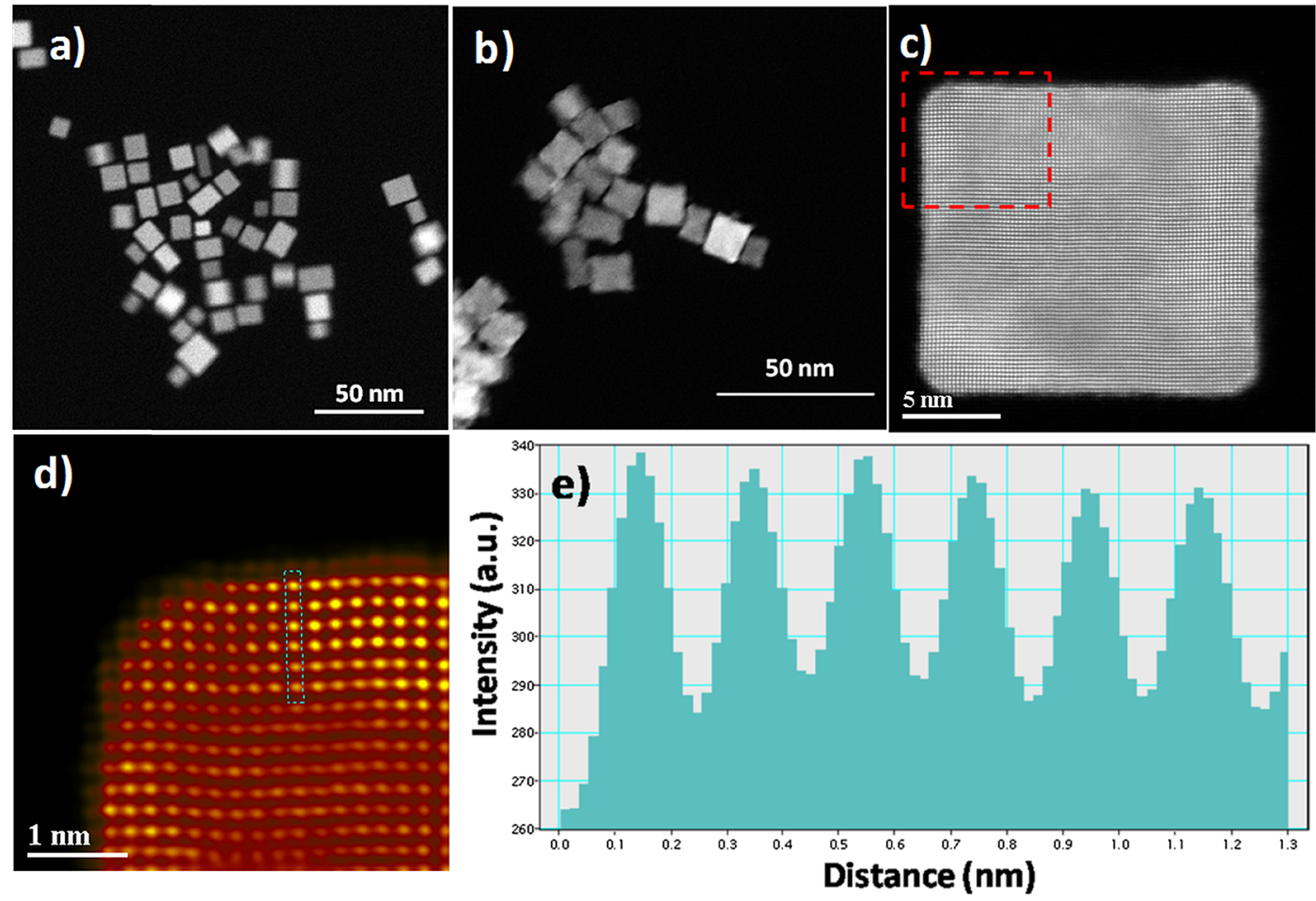

Figure 1. (a,b) STEM images of Pd and Pd@Pt core-shell nanocubes. (c) HAADF-STEM image of the individual Pd@Pt NCs. (d) High-resolution HAADF-STEM image taken from the region marked by a box in (c), revealing a Pt shell thickness of four atomic layers. (e) Intensity profile along the region marked in $(\mathrm{d})$ showing six atomic layers.

merely increasing the $\mathrm{pH}$ to induce the reduction power of ascorbic acid (AA), thereby blocking the galvanic reaction between $\mathrm{Ag}$ and $\mathrm{Au}^{3+}$, and to achieve conformal deposition of three to six atomic layers of $\mathrm{Au}$ shell on $\mathrm{Ag}$ nanocubes. However, such a method has not been investigated for other types of nanoparticles (NPs).

Several strategies have been reported to overcome the limitation for the enzyme immobilization and facilitate enhanced direct electron transfer (DET) process for metal NP-based biosensors. ${ }^{14}$ Pt NPs have been highly exploited for developing glucose sensors ${ }^{15}$ and human metabolite detection. ${ }^{16}$ Nevertheless, the high cost of Pt significantly limits its practical applications in biosensors. Therefore, many groups have attempted to create Pt-based bimetallic NCs because of the possibility to tune the Pt d-band structure, which correlates with the adsorption strength of the catalyst surface, that is, $\mathrm{Pt}$ (100) surface through the strain and ligand effects. ${ }^{6}$ Moreover, bimetallic Pd@Pt core-shell nanocubes with sharp corners and edges can significantly enhance the glucose oxidation activities. ${ }^{17} \mathrm{Pt} / \mathrm{Pd}$ alloy NPs exhibit enzyme-mimic activity that can actively catalyze the $\mathrm{H}_{2} \mathrm{O}_{2}$ reduction to detect $\mathrm{H}_{2} \mathrm{O}_{2}$ in various environments. ${ }^{18}$ Dumbbell-like $\mathrm{PtPd}-\mathrm{Fe}_{3} \mathrm{O}_{4}$ NPs exhibit an enhanced sensitivity relative to individual components for the continuous monitoring of $\mathrm{H}_{2} \mathrm{O}_{2}$ released from RAW264.7 cells. ${ }^{4,5}$ The enhancement in the activity is not only due to the alloy structure and composition but also due to the NP interface, which acts as a tuning factor to improve the catalytic activity of the biosensor. ${ }^{19}$ So far, there have been limited studies on glucose monitoring and the detection mechanism using Pd and Pt-based NCs as electrocatalytic materials.

Engineering the metal NP surface by organic coatings remarkably improves the biocatalytic activity and preserves the structure of glucose oxidase (GOx)..$^{20,21}$ Polymeric capping agents can facilitate the incorporation of enzymes on the NP surface without losing their activity, and the charge transfer between the electron donar [flavin adenine dinucleotide $(\mathrm{FAD})]$ and the surface of the electrode is more efficient in amperometric enzyme-based glucose biosensors. ${ }^{22,23}$ The immobilization of the enzymes on the solid support is the critical issue that strongly affects the biocatalytic activity and thus the sensitivity of the biosensor. ${ }^{14,24}$ Numerous materials have been developed such as block copolymers, ${ }^{25}$ conducting hydrogels, ${ }^{26}$ mesoporous silica, ${ }^{27}$ DNA scaffolds, ${ }^{28}$ bacteriophage $^{29}$ ionic liquids, ${ }^{30}$ metal-organic frameworks, ${ }^{31}$ carbon nanotubes (CNTs), ${ }^{32}$ halloysite nanotubes, ${ }^{33}$ and graphene oxide $(\mathrm{GO})^{34}$ as efficient templates for effective immobilization, preserving the biocatalytic activity of enzymes and facilitating DET. Among all, chitosan (CS) biopolymers hold promise for the immobilization of enzymes not only due to their active $-\mathrm{NH}_{3}{ }^{+} / \mathrm{NH}_{2}$ and $-\mathrm{OH}$ functional groups but also due to their high biocompatibility and low cost. ${ }^{35,36}$ In our previous study, we have shown that CS-stabilized silver nanowires show enhanced charge transfer and stability for electrochemical detection of glucose. ${ }^{20}$ The Pd@Pt core-shell nanocubes covered with CS provide synergizing activity of highly biocompatible CS and excellent electrocatalytic activity, high surface area, and superior binding affinity to biomolecules and, thus, are well-suited for the design of biosensors with high sensitivity and stability.

Herein, we report a facile, room-temperature route to synthesize Pd@Pt core-shell nanocubes with conformal deposition of ultrathin Pt shell (six atomic layers). The thickness of the Pt shells on the Pd nanocubes could be precisely tuned by simply varying the concentration of the $\mathrm{Pt}$ precursor. The key success of our synthesis strategy mainly 
relies on using AA for fast reduction of $\mathrm{Pt}$ atoms by increasing the $\mathrm{pH}$ of the reaction, which allows the control of the reduction kinetics. Moreover, the resultant Pd@Pt NCs are covered with biocompatible $\mathrm{CS}$ as an efficient covalent immobilization matrix for the enzymatic electrochemical detection of glucose. We demonstrate remarkably enhanced sensitivity and selectivity to detect glucose based on CS/Pd@Pt NC/GOx enzyme electrodes.

\section{RESULTS AND DISCUSSION}

A typical scanning transmission electron microscopy (STEM) image of the as-prepared Pd nanocubes and Pd@Pt nanocubes has an average size of $13 \mathrm{~nm}$, as shown in the STEM images, and a corresponding size distribution histogram, as shown in Figure S1. Figure 1a,b shows the STEM analysis of bare Pd nanocubes and Pd@Pt NCs revealing the conformal deposition of Pt layers of a-few-atomic-layer thickness onto the surface of each Pd nanocube. The high-angle annular dark-field STEM (HAADF-STEM) image of the individual Pd@Pt NCs shows a clear contrast between the Pd core and the subnanometer-thick Pt shell that exhibits a periodic lattice plane extending across the entire surface, suggesting the single-crystalline nature of the Pt shell (Figure 1c). These contrast variations between the Pd core and $\mathrm{Pt}$ shell can be due to the large difference in atomic numbers between $\mathrm{Pd}$ and Pt elements. ${ }^{37}$ Furthermore, the initial cubic shape of the Pd NCs remains the same, indicating the controlled layer-by-layer deposition of $\mathrm{Pt}$. The atomicresolution HAADF-STEM measurements for the resultant Pd@Pt NCs reveal six atomic layers of Pt shell (Figure 1d). Varying from 6 atomic layers to 10 and 20 layers is feasible by altering the amount of the $\mathrm{Pt}$ precursor solution from 0.1 to $0.25 \mathrm{~mL}$ and $0.5 \mathrm{~mL}$, respectively (Figure S2). The intensityprofiling analysis (Figure 1e) along the region shown in Figure $1 \mathrm{~d}$ further confirms the $\mathrm{Pd}$ core and the six atomic layers of $\mathrm{Pt}$ shell thickness. In addition, the energy-dispersive X-ray (EDX) elemental analysis and mapping (Figure S3) show the presence of Pd and Pt elements, and the color difference between the Pd core and Pt shell further confirms the homogeneous deposition of $\mathrm{Pt}$ on the surfaces of $\mathrm{Pd}$ nanocubes. The $\mathrm{Pt}$ atomic ratio was obtained using the inductively coupled plasma-atomic emission spectroscopy (ICP-AES) analysis as $7.05 \%$ of Pt in the resultant Pd@Pt NCs. Furthermore, X-ray photoelectron spectroscopy (XPS) measurements indicate a presence of both $\mathrm{Pd}$ and $\mathrm{Pt}$ elements with a Pt content of 7.15 wt \% (Figure S4), which is consistent with the ICP result.

Previous reports have shown that because of the relatively higher standard reduction potential of $\mathrm{PtCl}_{6}{ }^{2-} / \mathrm{Pt}[0.74 \mathrm{~V}$ vs rotation reversible hydrogen electrode (RHE)] than $\mathrm{PdCl}_{4} / \mathrm{Pd}$ (0.62 V vs RHE), the deposition of Pt on the Pd nanocubes undergoes a galvanic replacement reaction, leading to the formation of concave Pd@Pt core-shell NCs. ${ }^{38} \mathrm{Pt}$ atoms can be uniformly deposited by heterogeneous nucleation on the Pd cubes at higher temperatures $\left(180{ }^{\circ} \mathrm{C}\right)$, in which the homogeneous deposition with a well-defined shell thickness is largely determined by the difference in the $\mathrm{Pt}$ deposition rate $\left(R_{\text {dep }}\right)$ and the surface diffusion rate $\left(R_{\text {dif }}\right) \cdot{ }^{10,39}$ In our present synthesis strategy, the deposition was done using a relatively strong reducing agent (AA) at room temperature, allowing the reduction of $\mathrm{Pt}$ immediately and selectively at the corner sites of the Pd nanocubes because of the high-energy $\mathrm{Pd}\{100\}$ facets and then diffusing onto the side faces. Therefore, the homogeneous deposition largely depends on the $R_{\text {dif }}$. The $\mathrm{pH}$ of the reaction system is found to be critical for accelerating the surface diffusion of reduced Pt atoms for uniform deposition. The reduction power of reducing agents such as AA can be controlled by simply adjusting the $\mathrm{pH}$ value of the reaction solution, and thus, the reaction kinetics can be easily altered. ${ }^{13}$ When the $\mathrm{pH}$ of the reaction solution is adjusted to $\mathrm{pH}=11$, the surface diffusion of $\mathrm{Pt}$ atom is significantly enhanced, thereby creating the condition of $R_{\mathrm{dep}}<R_{\mathrm{dif}}$. Consequently, the reduced $\mathrm{Pt}$ atoms quickly diffuse onto the side faces to achieve a uniform Pt shell with controlled thickness. By contrast, relatively lower $\mathrm{pH}(\mathrm{pH}=4)$ under equivalent reaction conditions fails to produce uniform deposition. Interestingly, the resultant products are concave Pd@Pt core-shell NCs (Figure S5). These results clearly demonstrate that the $\mathrm{pH}$ of the reaction system plays a key role in modulating the reduction kinetics.

Fabrication of the enzyme electrode based on CS/Pd@Pt NC/GOx-glassy carbon electrode (GCE) comprises of three steps: preparation of the $\mathrm{Pd} @ \mathrm{Pt}$ core-shell NCs, surface functionalization with $\mathrm{CS}$, and covalent immobilization of GOx enzyme (Scheme 1). Initially, the NCs were prepared and then

Scheme 1. Schematic Displaying the Surface Modification of Pd@Pt NCs Using CS Biopolymer and the Covalent Immobilization of GOx to the CS by Reacting with GA to Cross-Link the Amino Group of CS and the FAD Site of GOx

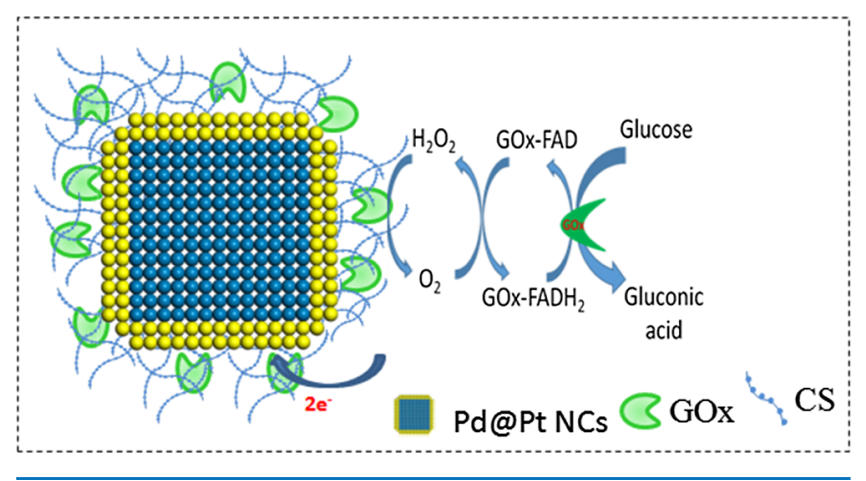

the CS biopolymer was used to functionalize through electrostatic interactions. After coating the NCs with the CS polymer, small CS layers encapsulated onto the NCs are formed, as can be seen in the transmission electron microscope (TEM) image shown in Figure 2a. The presence of amino groups in the highly biocompatible and hydrophilic CS permits covalent immobilization of GOx enzymes by reacting with the bifunctional cross-linker glutaraldehyde (GA). ${ }^{35,40}$ The glucosesensing principle of GOx-functionalized NCs is based on the catalytic oxidation of glucose into gluconic acid and $\mathrm{H}_{2} \mathrm{O}_{2}$ in the presence of oxygen via an enzymatic reaction (Scheme 1). The GOx enzyme contains its active cofactor FAD bound to its two identical $80 \mathrm{kDa}$ subunits. The efficient covalent immobilization of the GOx, the FAD cofactors being electrically contacted properly, and minimizing the electron-tunneling distance by bringing down the deeply buried FAD are all key factors. ${ }^{41,42}$ As a result, DET between the FAD center and the electrode surface is promoted and protection from losing the biocatalytic function improves the lifetime. ${ }^{25,43}$ Homogeneous dispersion of $\mathrm{Pd} @ \mathrm{Pt} \mathrm{NCs}$ in CS polymer catalyzes the electrochemical oxidation of the enzymatically liberated $\mathrm{H}_{2} \mathrm{O}_{2}$ and promotes the enhanced charge transfer, thereby resulting in 
a)

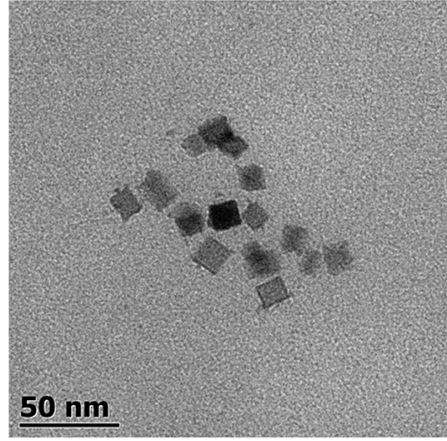

b)

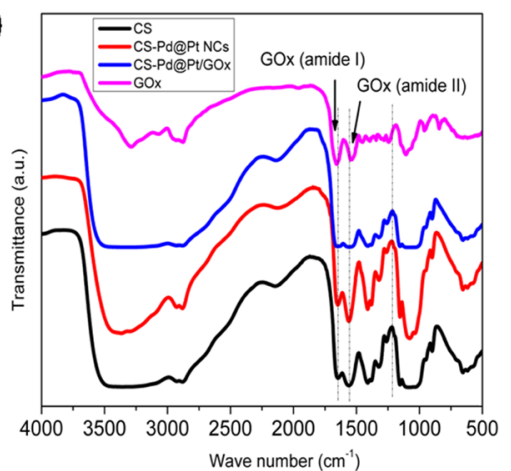

Figure 2. (a) TEM image of the CS-covered Pd@Pt NCs. (b) FT-IR spectra of the bare CS, CS/Pd@Pt NCs, CS/Pd@Pt NC/GOx, and native GOx.

the remarkable improvement in the sensitivity of the resultant biosensor.

The strategy for enzyme immobilization is the key factor that affects the biosensor performance by altering the charge transfer between the FAD center and the electrode surface, modulating the electron-tunneling distance and controlling the leaching effects. ${ }^{24,44}$ The CS contains an abundance of $-\mathrm{HN}_{3}{ }^{+}$/ $\mathrm{NH}_{2}$ and $-\mathrm{OH}$ functional groups. ${ }^{20}$ The $-\mathrm{NH}$-terminated surfaces were reacted with the bifunctional compound GA to cross-link the $-\mathrm{NH}_{2}$ groups of the GOx by covalent linkage. ${ }^{16,35}$ The $\mathrm{NH}_{2}$-terminated surface of the $\mathrm{CS}$ was covalently linked by forming $\mathrm{C}-\mathrm{N}$ bonds to the amino groups on the GOx by reacting with the two aldehyde groups on the GA. ${ }^{45}$ We confirmed the structural interaction and immobilization of GOx enzymes with the CS/Pd@Pt NCs using Fourier transform infrared (FT-IR) spectroscopy (Figure 2b). The GOx shows characteristic transmittance bands at 1658, 1542, and $1103 \mathrm{~cm}^{-1}$ associated with the amide I and amide II absorption bands of the proteins and the $\mathrm{C}-\mathrm{O}$ stretching vibration of GOx, respectively. ${ }^{46}$ From the comparison of the spectra, the following are evident: appearance of new peaks at $1643 \mathrm{~cm}^{-1}$ band for amide I ( $\mathrm{C}=\mathrm{O}$ stretching vibrations), at $1556 \mathrm{~cm}^{-1}$ for amide II (N-H bending vibrations), and bands at $1410 \mathrm{~cm}^{-1}$ for $-\mathrm{OH}$ bending vibrations upon $\mathrm{GOx}$ conjugation, suggesting the covalent binding through the $\mathrm{C}-$ $\mathrm{N}$ bonds and retaining the secondary structure of $\mathrm{GOx}{ }^{47}$ In addition, dynamic light scattering (DLS) and zeta potential measurements were recorded to characterize the functionalization process. Table S1 presents the results for the hydrodynamic diameter determined from DLS and the corresponding surface charge obtained from the zeta potential analysis. From the DLS, the bare Pd@Pt NCs show a diameter of 13 $\mathrm{nm}$, and this value increases to $17.3 \mathrm{~nm}$ after the CS coverage. At the same time, the surface charge of the bare Pd@Pt NCs inverts from a negative $-1.02 \mathrm{mV}$ to a positive value of 36.23 $\mathrm{mV}$, suggesting the increase in the size and stability upon CS coverage on the NCs. After the immobilization of GOx, the diameter further increases to $37.1 \mathrm{~nm}$, with a decrease in the zeta potential to $7.25 \mathrm{mV}$. The decrease in the surface charge is due to the interaction of $-\mathrm{NH}$ groups with the GOx enzyme through the GA molecules. ${ }^{40}$

Amperometric glucose detection using the fabricated electrode was performed using cyclic voltammetry (CV) and chronoamperometry measurements (CA). The CVs comparing the bare GCE, CS/Pd@Pt NC-GCE, and CS/Pd@Pt NC/ GOx-GCE in $\mathrm{N}_{2}$-saturated phosphate-buffered saline (PBS, $\mathrm{pH}$ 7.4) at a scan rate of $50 \mathrm{mV} \cdot \mathrm{s}^{-1}$ are shown in Figure 3. The

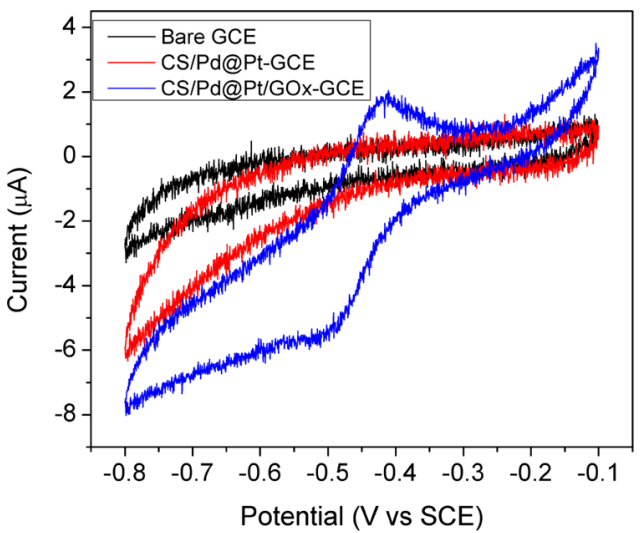

Figure 3. CVs of a bare GCE (black), CS/Pd@Pt NC-GCE (red), and CS/Pd@Pt NC/GOx-GCE (blue) in a $\mathrm{N}_{2}$-saturated PBS $(\mathrm{pH}=7)$ at a scan rate of $50 \mathrm{mV} \cdot \mathrm{s}^{-1}$.

results show that the bare GCE and CS/Pd@Pt NC-GCE exhibit no redox peaks in the potential range of interest. Nevertheless, the CV of the CS/Pd@Pt NC/GOx-GCE shows two well-defined redox peaks at cathodic $\left(E_{\mathrm{pc}}\right)$ and anodic $\left(E_{\mathrm{pa}}\right)$ peak potentials of -0.49 and $-0.42 \mathrm{~V}$, respectively. These redox peak potentials are close to the standard oxidation and reduction potentials of $\mathrm{FAD} / \mathrm{FADH}_{2}$ of GOx. ${ }^{43,46}$ This result indicates that our modified electrode can promote the DET between the electron donor (FAD) and the electrode surface.

The enzyme coverage density $\left(\Gamma, \mathrm{mol} / \mathrm{cm}^{2}\right)$ of the immobilized GOx onto the CS/Pd@Pt NC/GOx-modified electrode can be estimated by integrating the cathodic peak according to the following equation. ${ }^{48}$

$$
\Gamma=Q / n F A
$$

where $Q$ is the charge consumed in the redox reaction (obtained by integrating the anodic peak and dividing by the scan rate of $\left.50 \mathrm{mV} \cdot \mathrm{s}^{-1}\right), n$ is the number of electrons transferred (in this case, $n=2$ ), $F$ is the Faraday constant, and $A$ is the geometric area of the GCE $\left(0.07 \mathrm{~cm}^{2}\right)$. The estimated electroactive immobilized enzyme coverage on the electrode is $4.2 \times 10^{-8} \mathrm{~mol} / \mathrm{cm}^{2}$. This value is comparable to the previous report for covalent conjugation with AuNPs, which supported the M13 bacteriophage $\left(4.74 \times 10^{-8} \mathrm{~mol} / \mathrm{cm}^{2}\right),{ }^{29}$ and is higher than those for GOx immobilized onto $\mathrm{CS} /$ carbon nanodots $\left(8.78 \times 10^{-11} \mathrm{~mol} / \mathrm{cm}^{2}\right),{ }^{49}$ graphene oxide $\left(1.22 \times 10^{-10} \mathrm{~mol} /\right.$ $\left.\mathrm{cm}^{2}\right),{ }^{50} \mathrm{TiO}_{2}$ nanostructures $\left(2.57 \times 10^{-10} \mathrm{~mol} / \mathrm{cm}^{2}\right),{ }^{51}$ and boron-doped CNT $\left(1.94 \times 10^{-9} \mathrm{~mol} / \mathrm{cm}^{2}\right) .{ }^{52}$ 

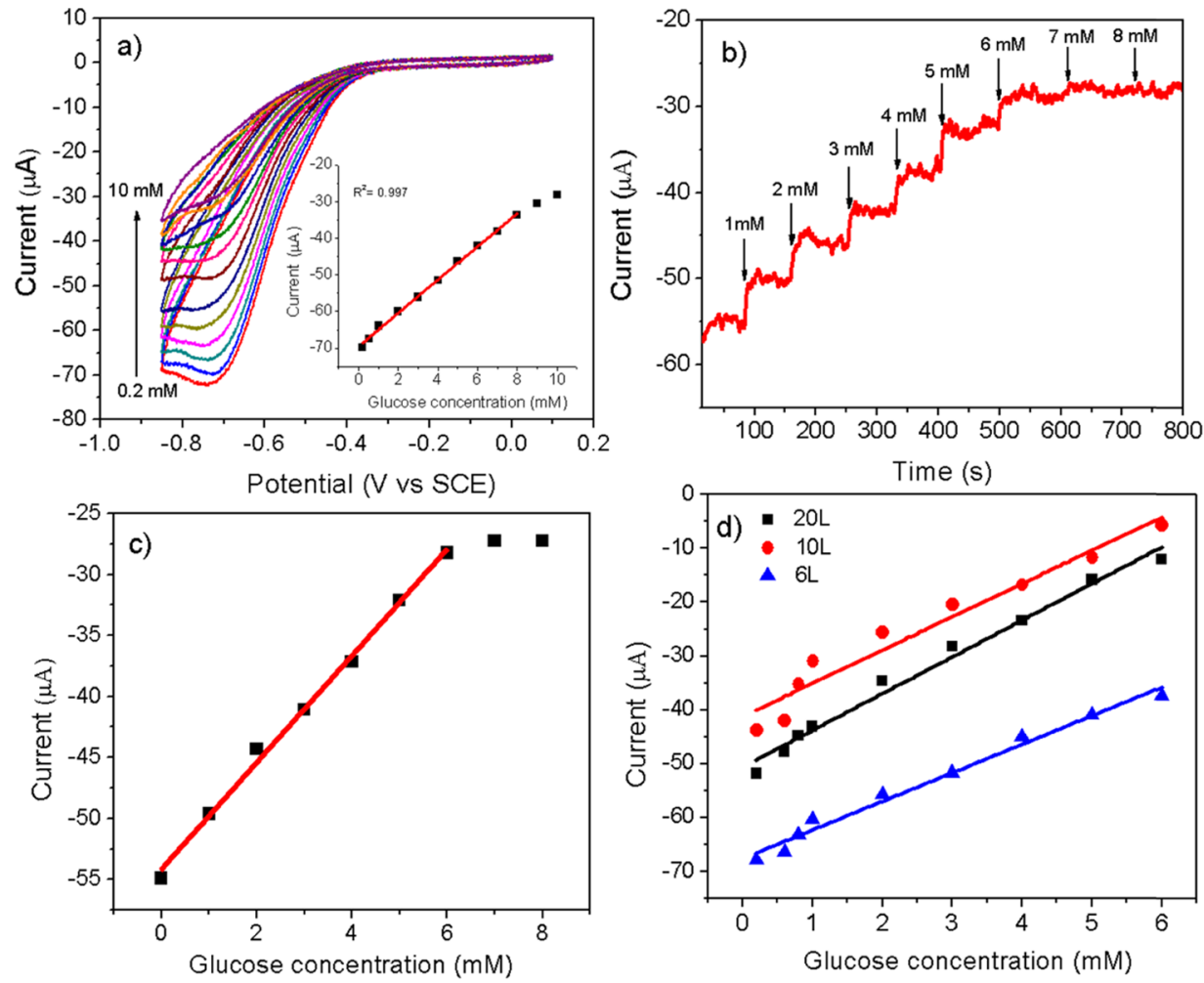

Figure 4. (a) CVs corresponding to the electrocatalyzed oxidation of different concentrations of glucose by the GOx-immobilized CS/Pd@Pt NC/ GOx-GCE. CVs were recorded in PBS $(0.1 \mathrm{M}, \mathrm{pH} 7.4)$ at a scan rate of $50 \mathrm{mV} \cdot \mathrm{s}^{-1}$. (b) Current-time response curves for the successive addition of glucose $(1-8 \mathrm{mM})$ at a fixed potential of $-0.65 \mathrm{~V}$. (c) Linear calibration curve corresponding to the amperometric response of the CS/Pd@Pt NC/ GOx-GCE in the presence of variable concentration of glucose. (d) Linear calibration curves corresponding to varying Pt shell thicknesses in the presence of different glucose concentrations.

Table 1. Comparison of the Analytical Performance of Different Nanomaterial-Based DETs for Electrochemical Sensing of Glucose $^{a}$

\begin{tabular}{|c|c|c|c|c|c|c|}
\hline biosensor & sensitivity $\left(\mu \mathrm{Acm}^{-2} \mathrm{mM}^{-1}\right)$ & response time $(\mathrm{s})$ & detection limit $(\mu \mathrm{M})$ & linear range $(\mathrm{mM})$ & $K_{\mathrm{m}}(\mathrm{mM})$ & ref \\
\hline $\mathrm{PtNW} / \mathrm{GOx}$ & 4.21 & & 83.3 & $0.083-182$ & 17.6 & 57 \\
\hline $\mathrm{DMIm} / \mathrm{Au}_{25} / \mathrm{GOx}$ & 0.21 & & & $1-6$ & 3.4 & 30 \\
\hline 1-D $\mathrm{TiO}_{2} / \mathrm{GOx}$ & 9.9 & $<5$ & & $0.2-1$ & 1.54 & 46 \\
\hline $\mathrm{PMA} /$ diamond/GOx & 0.006 & & 1 & $0-3$ & & 23 \\
\hline $\mathrm{GR} / \mathrm{CNT} / \mathrm{ZnO} \mathrm{NPs} / \mathrm{GO} \mathrm{x}$ & 5.36 & & 4.5 & $0.01-6.5$ & & 58 \\
\hline $\mathrm{CNT} / \mathrm{GOx}$ & 0.47 & & 4 & $1-30$ & & 59 \\
\hline $\mathrm{PtNPs} / \mathrm{GR} / \mathrm{CS} / \mathrm{GOx}$ & & & 0.6 & $0.2-5$ & & 53 \\
\hline CS/Pd@Pt NC/GOx & 6.82 & $<5$ & 0.2 & $1-6$ & 0.857 & this worl \\
\hline
\end{tabular}

${ }^{a} \mathrm{PtNW}$ : platinum nanowires; DMI: 1-decyl-3-methylimidazolium; $\mathrm{TiO}_{2}$ : titanium dioxide; PMA: poly(methacrylic acid); CNT: carbon nanotubes; ZnO: zinc oxide; PtNPs: platinum nanoparticles; GR: graphene.

To investigate the DET characteristics for the electrochemical detection of glucose, we recorded $\mathrm{CV}$ measurements in oxygen $\left(\mathrm{O}_{2}\right)$-saturated $0.1 \mathrm{M}$ PBS solution $(\mathrm{pH} 7.4)$ at a scan rate of $50 \mathrm{mV} \cdot \mathrm{s}^{-1}$ in the presence of different concentrations of glucose. Figure $4 \mathrm{a}$ shows the CVs along with the respective calibration plots (inset) corresponding to the bio-electrocatalytic reduction of successive addition of varying glucose concentrations. The reduction current gradually decreases upon the addition of glucose, exhibits a linear range between 1 and $6 \mathrm{mM}$, and saturates after $6 \mathrm{mM}$. It should be pointed out that without covering with the CS, the bare Pd@ Pt/GOx electrode exhibits a rapid reduction in the low glucose concentration range and reaches saturation quickly after $4 \mathrm{mM}$ (Figure S6). This result indicates that the CS polymer confers stability to the modified electrode and prevents inactivation of biocatalytic functions, which enables an enhanced performance for glucose detection. The sensitivity of the biosensor was measured using current-time $(i-t)$ curves at a constant potential of $-0.5 \mathrm{~V}$ versus saturated calomel electrode (SCE), which is shown in Figure $4 \mathrm{~b}$. The electrocatalytic current decreases upon successive addition of glucose and reaches a steady state with an average response time of $5 \mathrm{~s}$. Notably, we can see that the reduction current decreases and saturates above $6 \mathrm{mM}$.

A typical steady-state current as a function of glucose concentration (Figure 4c) shows a linear relationship in the 1$6 \mathrm{mM}$ range, thus suggesting that the sensor can be used in this concentration range for the continuous monitoring of glucose. The observed low linear range can be attributed to the combined effect of intrinsic peroxide activity catalyzed by the enzymatic release of $\mathrm{H}_{2} \mathrm{O}_{2}$ and the competitive oxygen consumption by glucose and $\mathrm{FADH}_{2}{ }^{46}$ The linear range here 

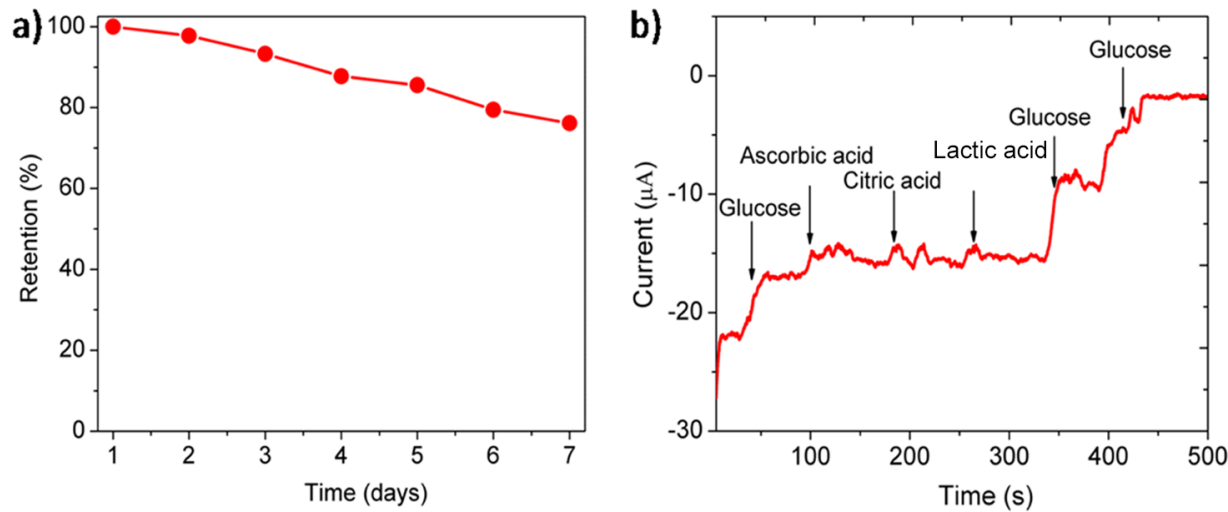

Figure 5. (a) Stability of the CS/Pd@Pt NC/GOx-GCE-modified electrode over a week-long storage period. (b) Amperometric response showing the effect of interfering substances ( $1 \mathrm{mM} \mathrm{AA}, \mathrm{CA}, \mathrm{LA}$, and glucose).

is slightly broader compared with that of Pt NPs supported on graphene and CS $(0-5 \mathrm{mM}){ }^{53}$ The sensitivity of the biosensor was calculated to be $6.82 \mu \mathrm{A} \mathrm{mM}{ }^{-1} \mathrm{~cm}^{-2}$, which is relatively higher than those of other reported glucose biosensors (Table 1 ). The sensitivity of our biosensor is less compared with the Pt NP-based enzyme electrode; the CS coating alters the reactivity of the Pd@Pt NCs and the conductivity, which significantly decreases the charge transport. The glucose concentration in the standard blood sample is approximately $4.89 \mathrm{mM}^{54}$ Our biosensor represents the linear relationship between the steadystate current as a function of the concentration of glucose (0.2$6 \mathrm{mM}$ ), which is higher than the average glucose level in the blood. We also carried out additional experiments to establish the detection limit of the biosensor, which was $0.2 \mu \mathrm{M}$ (Figure S7). This detection limit is also significantly better than those of the other recently reported biosensors (Table 1). The apparent Michaelis-Menten constant $\left(K_{\mathrm{m}}\right)$ was determined to evaluate the biological activity of the immobilized enzyme, which is estimated using Lineweaver-Burk equation as follows ${ }^{46}$

$$
\frac{1}{i_{\mathrm{ss}}}=\frac{K_{\mathrm{m}}^{\mathrm{app}}}{I_{\max }} \times \frac{1}{C}+\frac{1}{i_{\max }}
$$

where $i_{\mathrm{ss}}$ is the steady-state current after the addition of glucose, $i_{\max }$ is the maximum current under the saturated conditions, and $C$ is the bulk concentration of glucose. For a given glucose concentration, the calculated $K_{\mathrm{m}}$ is 0.58 , which is very small compared with those of the previously reported different nanostructure-based enzymatic glucose sensors presented in Table 1 . The relatively low value of $K_{\mathrm{m}}$ suggests a higher binding affinity of the immobilized GOx to the CS/Pd@Pt core-shell NC-based enzyme electrode and enzymatic activity. The excellent immobilized GOx affinity for glucose can be attributed to the high biocompatibility of CS, which preserves the biocatalytic function and the structure of GOx.

Recent studies have shown that the catalytic activity of the Pd@Pt core-shell NCs is dramatically altered by varying Pt shell thickness. ${ }^{8,10}$ This effect is attributed to the changes in the adsorption strength of the NCs through surface strain arising from the lattice mismatch between $\mathrm{Pd}$ and $\mathrm{Pt}$ and ligand effects. ${ }^{55}$ Therefore, we have examined the glucose-sensing performance with different $\mathrm{Pt}$ shell thicknesses. Figure $4 \mathrm{~d}$ shows the calibration plots for the detection of glucose with three different $\mathrm{Pt}$ shell thicknesses. The amperometric currents of the modified electrode are linearly dependent upon glucose concentration $(0-6 \mathrm{mM})$, which yields the sensitivity values of
6.82, 6.172, and $5.303 \mu \mathrm{A} \mathrm{cm}{ }^{-2} \mathrm{mM}^{-1}$ for 6, 10, and $20 \mathrm{Pt}$ atomic layers, respectively. These results demonstrate that the $\mathrm{Pd} @$ Pt NCs with a few atomic layers of Pt show a higher sensitivity to detect glucose, ascribed to the significantly higher catalytic activity of the Pt shells with fewer atomic layers.

To evaluate the stability of the biosensor, the electrode was stored at $4{ }^{\circ} \mathrm{C}$ in a $0.1 \mathrm{M} \mathrm{PBS}$ after use and tested every day for the current response for $1 \mathrm{mM}$ glucose for a 1 week period (Figure 5a). Our biosensor retained approximately $80 \%$ of its original response over 7 days, indicating excellent stability. The biosensor exhibits almost the same current with identical glucose concentrations, thus pointing out a good stability for practical applications. The biosensor is highly selective for the detection of glucose. As shown in Figure 5b, addition of different interference substances such as $1 \mathrm{mM}$ AA, citric acid (CA), uric acid (UA), and lactic acid (LA) results in negligible changes in the reduction current, whereas an apparent response in the current is observed for the subsequent addition of $1 \mathrm{mM}$ glucose, suggesting an excellent anti-interference ability of the Pd@Pt NC/GOx-based biosensor.

\section{CONCLUSIONS}

We have demonstrated a platform based on CS-covered Pd@Pt core-shell NCs for the sensitive electrochemical detection of glucose. The new room-temperature synthesis methodology is exploited for depositing ultrathin shells of $\mathrm{Pt}$ on the $\mathrm{Pd}$ nanocubes to obtain uniform $\mathrm{Pd} @ \mathrm{Pt}$ core-shell nanocubes. The covering of CS on the nanocubes allows the covalent linkage of GOx enzyme, which not only promotes DET but also confers stability and preserves biocatalytic functions of GOx. The biosensor here is capable of glucose detection with a high sensitivity of $6.82 \mu \mathrm{A} \mathrm{cm} \mathrm{mM}^{-1}$, a linear range of $1-6$ $\mathrm{mM}$, and a fast response time (approximately $5 \mathrm{~s}$ ). Moreover, the sensor shows high stability and specificity toward different interfering compounds. This biosensor platform offers many advantages such as low cost, superior electrocatalytic activity, significant enhancement in charge transport, and high sensitivity in detecting glucose.

\section{EXPERIMENTAL SECTION}

4.1. Materials. CS (medium molecular weight $M_{\mathrm{w}}=300$ $\mathrm{kDa}, 82 \%$ degree of deacetylation), sodium tetrachloropalladate (II) $\left(\mathrm{Na}_{2} \mathrm{PdCl}_{4}\right)$, potassium tetrachloroplatinate (II) $\left(\mathrm{K}_{2} \mathrm{PtCl}_{4}\right)$, potassium bromide ( $\mathrm{KBr}, 99 \%)$, poly(vinyl pyrrolidone) (PVP, $M_{\mathrm{w}}$ of 55000 ), L-ascorbic acid (AA, 99\%), GOx (from Aspergillus niger), D (+) glucose, CA $(\geq 99 \%)$, UA $(\geq 99 \%)$, 
LA ( $\geq 99 \%)$, sucrose ( $\geq 99 \%)$, and GA (50\%) were purchased from Sigma-Aldrich. Acetic acid (glacial, 99-100\%) was purchased from Merck. All reagents were of analytical grade and used as received. Ultrapure deionized (DI) water (resistivity of $18 \mathrm{M} \Omega \cdot \mathrm{cm}$ ) was used throughout all experiments.

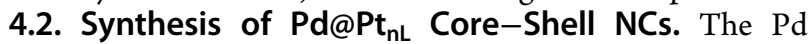
nanocube seeds with an average edge length of $13 \mathrm{~nm}$ were synthesized using a previously described method. ${ }^{56}$ In a typical synthesis, $15 \mathrm{~mL}$ of aqueous solution containing PVP, Lascorbic acid (60 mg), $\mathrm{KBr}(300 \mathrm{mg})$, and $\mathrm{Na}_{2} \mathrm{PdCl}_{4}(57 \mathrm{mg})$ was prepared and heated for $3 \mathrm{~h}$ at $80{ }^{\circ} \mathrm{C}$ under magnetic stirring. After that, the reaction mixture was cooled to room temperature, and then the resultant products were centrifuged at $12000 \mathrm{rpm}$ for $10 \mathrm{~min}$ and were washed with DI water to obtain Pd nanocubes. For the synthesis of Pd@Pt core-shell NCs, the Pd nanocubes were used as seeds for conformal deposition of Pt. In a typical procedure, $100 \mathrm{mg}$ of AA and 66.6 $\mathrm{mg}$ of PVP were dissolved in $15 \mathrm{~mL}$ of DI water in a vial, followed by adding $2.5 \mathrm{~mL}$ of aqueous suspension of $\mathrm{Pd}$ nanocubes, and the mixture was stirred well. After that, $0.5 \mathrm{~mL}$ of sodium hydroxide $(0.2 \mathrm{M})$ was added to increase the $\mathrm{pH}$ of the reaction to 11 . The mixture was magnetically stirred for 15 $\min$, and then, another aqueous solution $(0.1 \mathrm{~mL})$ containing $\mathrm{K}_{2} \mathrm{PtCl}_{4}(0.1 \mathrm{M})$ was added slowly to the vial to obtain Pd@Pt core-shell NCs. Varying the Pt atomic layers on the Pd cubes was done by simply changing the concentration of $\mathrm{K}_{2} \mathrm{PtCl}_{4}$ from 0.1 to $0.25 \mathrm{~mL}$ and $0.5 \mathrm{~mL}$.

4.3. Covering of Pd@Pt NCs with CS Biopolymer and Covalent Immobilization of GOx. The CS solution was obtained by dissolving $1 \mathrm{~g}$ of CS powder in a $1 \%$ acetic acid solution, as described in our previous work. ${ }^{20}$ The CS coating on the resultant NCs was performed by stirring $5 \mathrm{mg}$ of $\mathrm{Pd} @ \mathrm{Pt}$ nanocubes in $1 \mathrm{~mL}$ of CS solution (1 wt \%) for $30 \mathrm{~min}$. The covalent immobilization of GOx on the CS-covered Pd@Pt NCs was accomplished by reacting with $1 \mathrm{~mL}$ of GA (50\%), and the suspension was magnetically stirred for $2 \mathrm{~h}$, followed by the addition of $0.2 \mathrm{~mL}$ of GOx enzyme ( $40 \mathrm{mg} / \mathrm{mL}$ in PBS); the resultant solution was allowed to react overnight. The GOximmobilized CS/Pd@Pt NCs were then stored for the preparation of the enzyme electrode.

4.4. Preparation of the Enzyme Electrode. GCEs $(d=3$ $\mathrm{mm}$, homemade) were polished with $1.0,0.3$, and $0.05 \mu \mathrm{m}$ alumina powders to obtain a mirror surface. The GCE was rinsed thoroughly with DI water between each polishing step and sequential ultrasonication in a DI/acetone mixture and then dried with a nitrogen stream. After drying, $30 \mu \mathrm{L}$ of covalently immobilized GOx on the CS/Pd@Pt NCs was deposited on the surface of the cleaned GCE and left to dry at 4 ${ }^{\circ} \mathrm{C}$ for at least $4 \mathrm{~h}$. The fabricated modified enzyme electrodes were stored at $4{ }^{\circ} \mathrm{C}$ in a refrigerator under dry conditions when not in use.

4.5. Material Characterization. The morphology of the Pd nanocubes and Pd@Pt NCs was analyzed by TEM using a JEOL JEM-1010 instrument operated at $80 \mathrm{kV}$ by drop-casting the resultant $\mathrm{NCs}$ on the $\mathrm{Cu}$ grids and drying at room temperature. HAADF-STEM analyses were obtained using JEM ARM 200F equipment operated at an accelerating voltage of $200 \mathrm{kV}$. The covalent immobilization and the interaction of GOx with the CS/Pd@Pt NCs were identified using FT-IR spectroscopy using a Perkin Elmer spectrophotometer with an attenuated total reflection accessory in the range of 4000-650 $\mathrm{cm}^{-1}$. Electrochemical measurements were recorded on an electrochemical workstation (VoltaLab 40 PGZ 301). The DLS and zeta potential measurements were recorded using Nano ZS (Malvern).

4.6. Electrochemical Measurements. All electrochemical experiments were carried out using a VoltaLab 40 PGZ 301 electrochemical workstation with a conventional standard three-electrode cell. The homemade GCE used as the working electrode was cleaned well before and after each experiment. A platinum foil and an SCE were used as the auxiliary and the reference electrodes, respectively. The electrochemical glucosesensing measurements of the modified electrodes were recorded using $\mathrm{CV}$ and recorded in $0.1 \mathrm{M}$ PBS ( $\mathrm{pH}$ 7.4) at room temperature. The PBS buffer solution was purged with oxygen for at least $20 \mathrm{~min}$ before the measurements. The chronoamperometric measurements were recorded at a fixed potential of $-0.5 \mathrm{~V}$.

\section{ASSOCIATED CONTENT}

\section{Supporting Information}

The Supporting Information is available free of charge on the ACS Publications website at DOI: 10.1021/acsomega.7b00060.

STEM images, TEM images of different Pt shell thicknesses, EDX-elemental mapping analysis, XPS results, DLS and zeta potential analysis, and electrochemical response for low detection limits (PDF)

\section{AUTHOR INFORMATION}

\section{Corresponding Authors}

*E-mail: skumar@fata.unam.mx (S.K.K.).

*E-mail: resparza@fata.unam.mx (R.E.).

ORCID $\odot$

Siva Kumar Krishnan: 0000-0002-9672-9335

Notes

The authors declare no competing financial interest.

\section{ACKNOWLEDGMENTS}

This work was partially supported by the DGAPA postdoctoral fellowship, UNAM. The authors are grateful to Ma. Lourdes Palma Tirado (Campus UNAM Juriquilla, Qro) for TEM measurements.

\section{REFERENCES}

(1) Xia, Y.; Yang, X. Toward Cost-Effective and Sustainable Use of Precious Metals in Heterogeneous Catalysts. Acc. Chem. Res. 2017, 50, 450-454.

(2) Zhang, H.; Jin, M.; Xia, Y. Noble-Metal Nanocrystals with Concave Surfaces: Synthesis and Applications. Angew. Chem., Int. Ed. 2012, 51, 7656-7673.

(3) Zhang, L.; Roling, L. T.; Wang, X.; Vara, M.; Chi, M.; Liu, J.; Choi, S.-I.; Park, J.; Herron, J. A.; Xie, Z.; Mavrikakis, M.; Xia, Y. Platinum-based Nanocages with Subnanometer-Thick Walls and WellDefined, controllable Facets. Science 2015, 349, 412-416.

(4) Sun, X.; Guo, S.; Chung, C.-S.; Zhu, W.; Sun, S. A Sensitive $\mathrm{H}_{2} \mathrm{O}_{2}$ Assay based on Dumbbell-Like PtPd- $\mathrm{Fe}_{3} \mathrm{O}_{4}$ Nanoparticles. Adv. Mater. 2013, 25, 132-136.

(5) Sun, X.; Guo, S.; Liu, Y.; Sun, S. Dumbbell-Like PtPd- $-\mathrm{Fe}_{2} \mathrm{O}_{3}$ Nanoparticles for Enhanced Electrochemical Detection of $\mathrm{H}_{2} \mathrm{O}_{2}$. Nano Lett. 2012, 12, 4859-4863.

(6) Hossain, M. F.; Park, J. Y. Amperometric Glucose Biosensor Based on Pt-Pd Nanoparticles Supported by Reduced Graphene Oxide and Integrated with Glucose Oxidase. Electroanalysis 2014, 26, 940951. 
(7) Li, X.; Liu, Y.; Hemminger, J. C.; Penner, R. M. Catalytically Activated Palladium@Platinum Nanowires for Accelerated Hydrogen Gas Detection. ACS Nano 2015, 9, 3215-3225.

(8) Zhao, X.; Chen, S.; Fang, Z.; Ding, J.; Sang, W.; Wang, Y.; Zhao, J.; Peng, Z.; Zeng, J. Octahedral Pd@Pt1.8Ni Core-Shell Nanocrystals with Ultrathin PtNi Alloy Shells as Active Catalysts for Oxygen Reduction Reaction. J. Am. Chem. Soc. 2015, 137, 2804-2807.

(9) Toyoda, E.; Jinnouchi, R.; Ohsuna, T.; Hatanaka, T.; Aizawa, T.; Otani, S.; Kido, Y.; Morimoto, Y. Catalytic Activity of Pt/TaB2(0001) for the Oxygen Reduction Reaction. Angew. Chem., Int. Ed. 2013, 52, 4137-4140.

(10) Xie, S.; Choi, S.; Lu, N.; Roling, L. T.; Herron, J. A.; Zhang, L.; Park, J.; Wang, J.; Kim, M. J.; Xie, Z.; Mavrikakis, M.; Xia, Y. Atomic Layer-by-Layer Deposition of Pt on Pd Nanocubes for Catalysts with Enhanced Activity and Durability toward Oxygen Reduction. Nano Lett. 2014, 14, 3570-3576.

(11) Xia, Y.; Gilroy, K. D.; Peng, H.-C.; Xia, X. Seed-Mediated Growth of Colloidal Metal Nanocrystals. Angew. Chem., Int. Ed. 2016, 56, 60-95.

(12) Peng, Z.; Yang, H. Designer Platinum Nanoparticles: Control of Shape, Composition in Alloy, Nanostructure and Electrocatalytic Property. Nano Today 2009, 4, 143-164.

(13) Yang, Y.; Liu, J.; Fu, Z.-W.; Qin, D. Galvanic Replacement-Free Deposition of $\mathrm{Au}$ on $\mathrm{Ag}$ for Core-Shell Nanocubes with Enhanced Chemical Stability and SERS Activity. J. Am. Chem. Soc. 2014, 136, $8153-8156$.

(14) Tran, D. N.; Balkus, K. J. Perspective of Recent Progress in Immobilization of Enzymes. ACS Catal. 2011, 1, 956-968.

(15) Zhai, D.; Liu, B.; Shi, Y.; Pan, L.; Wang, Y.; Li, W.; Zhang, R.; Yu, G. Highly Sensitive Glucose Sensor Based on Pt Nanoparticle/ Polyaniline Hydrogel Heterostructure. ACS Nano 2013, 7, 35403546.

(16) Li, L.; Wang, Y.; Pan, L.; Shi, Y.; Cheng, W.; Shi, Y.; Yu, G. A Nanostructured Conductive Hydrogels-Based Biosensor Platform for Human Metabolite Detection. Nano Lett. 2015, 15, 1146-1151.

(17) Ye, J.-S.; Hong, B.-D.; Wu, Y.-S.; Chen, H.-R.; Lee, C.-L. Heterostructured Palladium-Platinum Core-Shell Nanocubes for Use in a Nonenzymatic Amperometric Glucose Sensor. Microchim. Acta 2016, 183, 3311-3320.

(18) Wang, H.; Bo, X.; Bai, J.; Wang, L.; Guo, L. Electrochemical Applications of Platinum-Palladium Alloy Nanoparticles/large Mesoporous Carbon. J. Electroanal. Chem. 2011, 662, 281-287.

(19) Lee, Y.; Garcia, M. A.; Frey Huls, N. A. F.; Sun, S. Synthetic Tuning of the Catalytic Properties of $\mathrm{Au}-\mathrm{Fe}_{3} \mathrm{O}_{4}$ Nanoparticles. Angew. Chem., Int. Ed. 2010, 49, 1271-1274.

(20) Kumar-Krishnan, S.; Chakaravarthy, S.; Hernandez-Rangel, A.; Prokhorov, E.; Luna-Bárcenas, G.; Esparza, R.; Meyyappan, M. Chitosan Supported Silver Nanowires as a Platform for Direct Electrochemistry and Highly Sensitive Electrochemical Glucose Biosensing. RSC Adv. 2016, 6, 20102-20108.

(21) Tsujimura, S.; Murata, K.; Akatsuka, W. Exceptionally High Glucose Current on a Hierarchically Structured Porous Carbon Electrode with "Wired" Flavin Adenine Dinucleotide-Dependent Glucose Dehydrogenase. J. Am. Chem. Soc. 2014, 136, 14432-14437. (22) Hung, A.; Mwenifumbo, S.; Mager, M.; Kuna, J. J.; Stellacci, F.; Yarovsky, I.; Stevens, M. M. Ordering Surfaces on the Nanoscale: Implications for Protein Adsorption. J. Am. Chem. Soc. 2011, 133, $1438-1450$.

(23) Reitinger, A. A.; Hutter, N. A.; Donner, A.; Steenackers, M.; Williams, O. A.; Stutzmann, M.; Jordan, R; Garrido, J. A. Functional Polymer Brushes on Diamond as a Platform for Immobilization and Electrical Wiring of Biomolecules. Adv. Funct. Mater. 2013, 23, 29792986.

(24) Mateo, C.; Palomo, J. M.; Fernandez-Lorente, G.; Guisan, J. M.; Fernandez-Lafuente, R. Improvement of Enzyme Activity, Stability and Selectivity via Immobilization Techniques. Enzyme Microb. Technol. 2007, 40, 1451-1463.

(25) Lee, J.; Ahn, H.; Choi, I.; Boese, M.; Park, M. J. Enhanced Charge Transport in Enzyme-Wired Organometallic Block Copoly- mers for Bioenergy and Biosensors. Macromolecules 2012, 45, 31213128.

(26) Li, L.; Shi, Y.; Pan, L.; Shi, Y.; Yu, G. Rational Design and Applications of Conducting Polymer Hydrogels as Electrochemical Biosensors. J. Mater. Chem. B 2015, 3, 2920-2930.

(27) Luckarift, H. R.; Spain, J. C.; Naik, R. R.; Stone, M. O. Enzyme Immobilization in a Biomimetic Silica Support. Nat. Biotechnol. 2004, 22, 211-213.

(28) Frasconi, M.; Heyman, A.; Medalsy, I.; Porath, D.; Mazzei, F.; Shoseyov, O. Wiring of Redox Enzymes on Three Dimensional SelfAssembled Molecular Scaffold. Langmuir 2011, 27, 12606-12613.

(29) Blaik, R. A.; Lan, E.; Huang, Y.; Dunn, B. Gold-Coated M13 Bacteriophage as a Template for Glucose Oxidase Biofuel Cells with Direct Electron Transfer. ACS Nano 2016, 10, 324-332.

(30) Kwak, K.; Kumar, S. S.; Pyo, K.; Lee, D. Ionic Liquid of a Gold Nanocluster: A Versatile Matrix for Electrochemical Biosensors. ACS Nano 2014, 8, 671-679.

(31) Wu, X.; Ge, J.; Yang, C.; Hou, M.; Liu, Z. Facile synthesis of multiple enzyme-containing metal-organic frameworks in a biomolecule-friendly environment. Chem. Commun. 2015, 51, 13408-13411.

(32) Lyons, M. E. G.; Keeley, G. P. Immobilized Enzyme-SingleWall Carbon Nanotube Composites for Amperometric Glucose Detection at a Very Low Applied Potential. Chem. Commun. 2008, 2529-2531.

(33) Chao, C.; Liu, J.; Wang, J.; Zhang, Y.; Zhang, B.; Zhang, Y.; Xiang, X.; Chen, R. Surface Modification of Halloysite Nanotubes with Dopamine for Enzyme Immobilization. ACS Appl. Mater. Interfaces 2013, 5, 10559-10564.

(34) Zhang, J.; Zhang, F.; Yang, H.; Huang, X.; Liu, H.; Zhang, J.; Guo, S. Graphene Oxide as a Matrix for Enzyme Immobilization. Langmuir 2010, 26, 6083-6085.

(35) Narang, J.; Pundir, C. S. Construction of a Triglyceride Amperometric Biosensor Based on Chitosan-ZnO Nanocomposite Film. Int. J. Biol. Macromol. 2011, 49, 707-715.

(36) Zhao, C.; Meng, Y.; Shao, C.; Wan, L.; Jiao, K. Unadulterated Glucose Biosensor Based on Direct Electron Transfer of Glucose Oxidase Encapsulated Chitosan Modified Glassy Carbon Electrode. Electroanalysis 2008, 20, 520-526.

(37) Wang, X.; Choi, S.-I.; Roling, L. T.; Luo, M.; Ma, C.; Zhang, L.; Chi, M.; Liu, J.; Xie, Z.; Herron, J. A.; Mavrikakis, M.; Xia, Y. Palladium-Platinum Core-Shell Icosahedra with Substantially Enhanced Activity and Durability towards Oxygen Reduction. Nat. Commun. 2015, 6, 7594.

(38) Zhang, H.; Jin, M.; Wang, J.; Li, W.; Camargo, P. H. C.; Kim, M. J.; Yang, D.; Xie, Z.; Xia, Y. Synthesis of Pd-Pt Bimetallic Nanocrystals with a Concave Structure through a Bromide-Induced Galvanic Replacement Reaction. J. Am. Chem. Soc. 2011, 133, 60786089.

(39) Xia, X.; Xie, S.; Liu, M.; Peng, H.-C.; Lu, N.; Wang, J.; Kim, M. J.; Xia, Y. On the Role of Surface Diffusion in Determining the Shape or Morphology of Noble-Metal Nanocrystals. Proc. Natl. Acad. Sci. U.S.A. 2013, 110, 6669-6673.

(40) Ma, X.; Jannasch, A.; Albrecht, U.-R.; Hahn, K.; Miguel-López, A.; Schäffer, E.; Sánchez, S. Enzyme-Powered Hollow Mesoporous Janus Nanomotors. Nano Lett. 2015, 15, 7043-7050.

(41) Xiao, Y.; Patolsky, F.; Katz, E.; Hainfeld, J. F.; Willner, I. "Plugging into Enzymes": Nanowiring of Redox Enzymes by a Gold Nanoparticle. Science 2003, 299, 1877-1881.

(42) Yehezkeli, O.; Tel-Vered, R.; Raichlin, S.; Willner, I. NanoEngineered Flavin-Dependent Glucose Dehydrogenase/Gold Nanoparticle-Modified Electrodes for Glucose Sensing and Biofuel Cell Applications. ACS Nano 2011, 5, 2385-2391.

(43) Bai, Y.-F.; Xu, T.-B.; Luong, J. H. T.; Cui, H.-F. Direct Electron Transfer of Glucose Oxidase-Boron Doped Diamond Interface: A New Solution for a Classical Problem. Anal. Chem. 2014, 86, 4910-4918.

(44) Hernandez, K.; Fernandez-lafuente, R. Control of Protein Immobilization: Coupling Immobilization and Site-Directed Mutagenesis to Improve Biocatalyst or Biosensor Performance. Enzyme Microb. Technol. 2011, 48, 107-122. 
(45) Barbosa, O.; Ortiz, C.; Berenguer-Murcia, Á.; Torres, R.; Rodrigues, R. C.; Fernandez-Lafuente, R. Glutaraldehyde in BioCatalysts Design: A Useful Crosslinker and a Versatile Tool in Enzyme Immobilization. RSC Adv. 2014, 4, 1583-1600.

(46) Si, P.; Ding, S.; Yuan, J.; Lou, X. W.; Kim, D.-H. Hierarchically Structured One-Dimensional $\mathrm{TiO}_{2}$ for Protein Immobilization, Direct Electrochemistry, and Mediator-Free Glucose Sensing. ACS Nano 2011, 5, 7617-7626.

(47) Tang, J.; Wang, Y.; Li, J.; Da, P.; Geng, J.; Zheng, G. Sensitive Enzymatic Glucose Detection by $\mathrm{TiO}_{2}$ Nanowire Photoelectrochemical Biosensors. J. Mater. Chem. A 2014, 2, 6153-6157.

(48) Rusling, J. F.; Forster, R. J. Electrochemical Catalysis with Redox Polymer and Polyion-Protein Films. J. Colloid Interface Sci. 2003, 262, $1-15$.

(49) Sheng, M.; Gao, Y.; Sun, J.; Gao, F. Carbon NanodotsChitosan Composite Film: A Platform for Protein Immobilization, Direct Electrochemistry and Bioelectrocatalysis. Biosens. Bioelectron. 2014, 58, 351-358.

(50) Unnikrishnan, B.; Palanisamy, S.; Chen, S.-M. A Simple Electrochemical Approach to Fabricate a Glucose Biosensor Based on Graphene-Glucose Oxidase Biocomposite. Biosens. Bioelectron. 2013, 39, 70-75.

(51) Bao, S.-J.; Li, C. M.; Zang, J.-F.; Cui, X.-Q.; Qiao, Y.; Guo, J. New Nanostructured $\mathrm{TiO}_{2}$ for Direct Electrochemistry and Glucose Sensor Applications. Adv. Funct. Mater. 2008, 18, 591-599.

(52) Deng, C.; Chen, J.; Chen, X.; Xiao, C.; Nie, L.; Yao, S. Direct Electrochemistry of Glucose Oxidase and Biosensing for Glucose Based on Boron-Doped Carbon Nanotubes Modified Electrode. Biosens. Bioelectron. 2008, 23, 1272-1277.

(53) Wu, H.; Wang, J.; Kang, X.; Wang, C.; Wang, D.; Liu, J.; Aksay, I. A.; Lin, Y. Glucose Biosensor Based on Immobilization of Glucose Oxidase in Platinum Nanoparticles/Graphene/Chitosan Nanocomposite Film. Talanta 2009, 80, 403-406.

(54) Song, Y.; Qu, K.; Zhao, C.; Ren, J.; Qu, X. Graphene Oxide: Intrinsic Peroxidase Catalytic Activity and Its Application to Glucose Detection. Adv. Mater. 2010, 22, 2206-2210.

(55) Kitchin, J. R.; Nørskov, J. K.; Barteau, M. A.; Chen, J. G. Role of Strain and Ligand Effects in the Modification of the Electronic and Chemical Properties of Bimetallic Surfaces. Phys. Rev. Lett. 2004, 93, 4-7.

(56) Li, G.; Kobayashi, H.; Taylor, J. M.; Ikeda, R.; Kubota, Y.; Kato, K.; Takata, M.; Yamamoto, T.; Toh, S.; Matsumura, S.; Kitagawa, H. Hydrogen Storage in Pd Nanocrystals Covered with a Metal-Organic Framework. Nat. Mater. 2014, 13, 802-806.

(57) Wang, Y.; Zhu, Y.; Chen, J.; Zeng, Y. Amperometric Biosensor Based on 3D Ordered Freestanding Porous Pt Nanowire Array Electrode. Nanoscale 2012, 4, 6025-6031.

(58) Hwa, K.-Y.; Subramani, B. Synthesis of Zinc Oxide Nanoparticles on Graphene-Carbon Nanotube Hybrid for Glucose Biosensor Applications. Biosens. Bioelectron. 2014, 62, 127-133.

(59) Jose, M. V.; Marx, S.; Murata, H.; Koepsel, R. R.; Russell, A. J. Direct Electron Transfer in a Mediator-Free Glucose Oxidase-Based Carbon Nanotube-Coated Biosensor. Carbon 2012, 50, 4010-4020. 